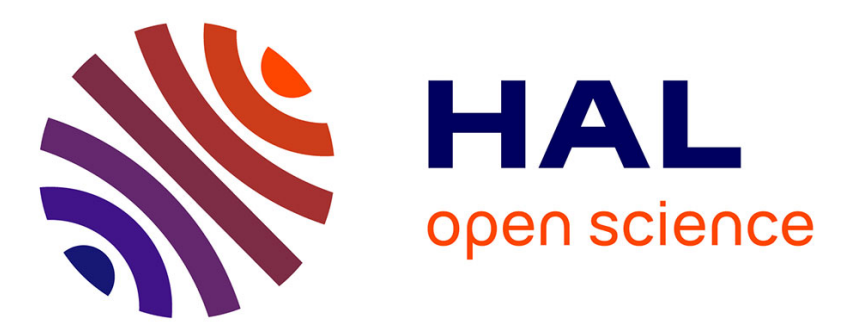

\title{
Tracking control for an ellipsoidal submarine driven by Kirchhoff's laws
}

Thomas Chambrion, Mario Sigalotti

\section{To cite this version:}

Thomas Chambrion, Mario Sigalotti. Tracking control for an ellipsoidal submarine driven by Kirchhoff's laws. IEEE Transactions on Automatic Control, 2008, 53 (1), pp.339-349. 10.1109/TAC.2007.914958 . hal-00097159

\section{HAL Id: hal-00097159 \\ https://hal.science/hal-00097159}

Submitted on 21 Sep 2006

HAL is a multi-disciplinary open access archive for the deposit and dissemination of scientific research documents, whether they are published or not. The documents may come from teaching and research institutions in France or abroad, or from public or private research centers.
L'archive ouverte pluridisciplinaire HAL, est destinée au dépôt et à la diffusion de documents scientifiques de niveau recherche, publiés ou non, émanant des établissements d'enseignement et de recherche français ou étrangers, des laboratoires publics ou privés. 


\title{
Tracking control for an ellipsoidal submarine driven by Kirchhoff's laws
}

\author{
Thomas Chambrion and Mario Sigalotti \\ Institut Elie Cartan UMR 7502, Nancy-Université/CNRS/INRIA \\ POB 239, Vandouvre-lès-Nancy 54506, France \\ Thomas.Chambrion@iecn.u-nancy.fr, mario.sigalotti@inria.fr
}

\begin{abstract}
In this paper, we study the control of an ellipsoid immersed in an infinite volume of ideal fluid. The dynamics of the uncontrolled body are given by Kirchhoff's laws. By adopting a backstepping viewpoint, we prove that the position and the attitude of the solid can be forced to approximatively follow any given path, using three controls (two controls for the direction and one control for the velocity). Moreover, we prove that the controlled mechanical system (which includes the momenta) is completely controllable in arbitrary small time.
\end{abstract}

Keywords - inviscid fluid, underactuated underwater vehicles, Kirchhoff's equations, output tracking, exact controllability, fast oscillating controls.

\section{Introduction}

Control of autonomous unmanned underwater vehicles has gained an increasing interest in the recent past years. Many problems concentrate on the control of very manoeuvrable robots. Such devices are easy to handle but usually very slow. By comparison, relatively few is known about the effective control of standard submarines, which are harder to handle and much quicker. In this paper, we establish the controllability of such a submarine driven by two directional controls (turn left/right and turn up/down) and one velocity control (back/forward).

\subsection{Physical context}

We model a submarine by a homogeneous neutrally buoyant ellipsoid immersed in an infinite volume (the entire space $\mathbf{R}^{3}$ ) of an inviscid incompressible fluid in irrotational motion. Due to the potential nature of the flow, the state of the system is fully determined by a finite set of real variables. Actually the system can be described in terms of impulse rather than in terms of momentum as could be expected. The impulse corresponds to the finite part of the momentum. Denoting by $(\omega, v)$ the standard (angular and linear) velocity of the ellipsoid with respect to a body-fixed coordinate frame, the corresponding impulse $(\Pi, P)$ can be expressed in the following way:

$$
\left(\begin{array}{c}
\Pi \\
P
\end{array}\right)=\left(\begin{array}{cc}
J & D \\
D^{t} & M
\end{array}\right)\left(\begin{array}{c}
\omega \\
v
\end{array}\right)
$$

where the 6-by-6 symmetric matrix $\mathcal{M}=\left(\begin{array}{cc}J & D \\ D^{t} & M\end{array}\right)$ is decomposed in 3-by-3 blocks. $J$ is the inertia matrix of the ellipsoid, $M$ corresponds to the mass of the body plus the added mass due to 
the action of the fluid on the body, and $D$ contains the coupling terms. If the axis of the coordinate frame coincide with those of the submarine, then $J$ and $M$ are diagonal and $D$ is equal to zero. We denote the diagonal entries of $M$ and $J$, respectively, by $M_{1}, M_{2}, M_{3}$ and $J_{1}, J_{2}, J_{3}$. The dynamics of the system are governed by the Kirchhoff equations (see [14] for details)

$$
\left\{\begin{array}{l}
\frac{d \Pi}{d t}=\Pi \times \omega+P \times v+\mathcal{T} \\
\frac{d P}{d t}=P \times \omega+\mathcal{F}
\end{array}\right.
$$

where $\times$ denotes the usual cross product in $\mathbf{R}^{3}$, while $\mathcal{T}$ and $\mathcal{F}$ denote, respectively, the external torque and the external force applied to the body. To help the reader distinguish between the different systems and subsystems considered in the paper, we also call "system $(\Pi, P)$ " the control system (2).

The assumptions we make on $\mathcal{T}$ and $\mathcal{F}$ are that two coordinates of $\mathcal{T}$ and one of $\mathcal{F}$ are tuned by the controller, in the following way,

$$
\mathcal{T}=\left(\begin{array}{c}
u_{1} \\
u_{2} \\
0
\end{array}\right), \quad \mathcal{F}=\left(\begin{array}{c}
0 \\
0 \\
u_{3}
\end{array}\right), \quad u_{1}, u_{2}, u_{3} \in \mathbf{R} .
$$

In the coordinate frame in which $\Pi$ and $P$ are expressed, the submarine is identified with an elipsoid $\Sigma \subset \mathbf{R}^{3}$. In a fixed reference frame, the submarine fills at time $t$ the subset $\Sigma(t)=r(t)+$ $A(t) \Sigma$, where $r$ and $A$ are, respectively, the position and the attitude of the submarine. The equations for $r$ and $A$ can be written in terms of $(\omega, v)$ using the classical formulas

$$
\frac{d A}{d t}=A S(\omega), \quad \frac{d r}{d t}=A v,
$$

where $S: \mathbf{R}^{3} \rightarrow$ so(3) is the linear bijection which associates to each vector $x \in \mathbf{R}^{3}$ the antisymmetric 3 -by-3 matrix $S(x)$ such that $x \times y=S(x) y$ for any $y$ in $\mathbf{R}^{3}$. We will speak of "system $(A, \Pi, r, P)$ " to refer to the control system (2),(4).

\subsection{Statement of the main results}

The dynamics of (2), i.e., the control of the momenta of the submarine (seen from the perspective of the solid), have been thoroughly studied (see for instance $[2,4,5,15,17]$ ). However, up to our knowledge, fewer is known about the extended control system $(A, \Pi, r, P)$ which describes the dynamics of the solid (states and momenta). Some optimal-control results of a planar version of system $(A, \Pi, r, P)$ can be found in $[8,9]$. Reconfiguaration algorithms (for initial and final conditions at velocity zero) are proposed in [7].

Our attention will be focused on the following two properties.

Definition 1.1 The system $(A, \Pi, r, P)$ is called exactly controllable if for every choice of an initial and a final condition $\left(A_{0}, \Pi_{0}, r_{0}, P_{0}\right)$ and $\left(A_{f}, \Pi_{f}, r_{f}, P_{f}\right)$, for every strictly positive real $T$, there exists a measurable bounded control $u=\left(u_{1}, u_{2}, u_{3}\right):[0, T] \rightarrow \mathbf{R}^{3}$ steering system $(A, \Pi, r, P)$ from $\left(A_{0}, \Pi_{0}, r_{0}, P_{0}\right)$ to $\left(A_{f}, \Pi_{f}, r_{f}, P_{f}\right)$.

Definition 1.2 We say that system $(A, \Pi, r, P)$ is state-trackable if for every smooth trajectory $(\bar{A}, \bar{r}):[0, T] \rightarrow S O(3) \times \mathbf{R}^{3}$, for every $\Pi_{0}, P_{0} \in \mathbf{R}^{3}$, and for every strictly positive tolerance $\epsilon$, there exists a measurable bounded control $u=\left(u_{1}, u_{2}, u_{3}\right):[0, T] \rightarrow \mathbf{R}^{3}$ such that the corresponding trajectory $t \mapsto(A(t), \Pi(t), r(t), P(t))$ with initial condition $(A(0), \Pi(0), r(0), P(0))=\left(\bar{A}(0), \Pi_{0}, \bar{r}(0), P_{0}\right)$ verifies the tracking condition: for every time $t$ in $[0, T],\|\bar{A}(t)-A(t)\| \mid<\epsilon$ and $\|\bar{r}(t)-r(t)\|<\epsilon$. 
Here above and in the following ||$.||$ denotes the usual Euclidean norm in $\mathbf{R}^{3},|||| \mid$. the induced norm on the space of 3-by-3 matrices, and the word smooth is used as a synonymous of belonging to the class $\mathcal{C}^{\infty}$.

It can be worth remarking that no a-priori causality relation can be established between trackability and exact controllability. Actually, tracking capabilities guarantee controllability just in the coordinates on which tracking is feasible. Reversely, there is no reason why a control system which is exactly controllable should admit coordinate submanifolds on which tracking is possible. Think, for instance, at the system $\dot{\theta}=1+u^{2}, u \in \mathbf{R}$, with $\theta \in \mathcal{S}^{1}=\mathbf{R} / \mathbf{Z}$.

The main goal of the paper is to prove the following results.

Theorem 1.3 Let $\left(J_{1}-J_{2}\right)^{2}+\left(M_{1}-M_{2}\right)^{2} \neq 0$. Then system $(A, \Pi, r, P)$ is exactly controllable.

Theorem 1.4 Let $\left(J_{1}-J_{2}\right)^{2}+\left(M_{1}-M_{2}\right)^{2} \neq 0$. Then system $(A, \Pi, r, P)$ is state-trackable.

\subsection{Content of the paper}

The paper is organized as follows. In Section 2 we start by recalling the precise continuos dependence result for ordinary differential equations which plays a fundamental role in our construction.

Section 3 presents the tracking construction in the case in which $J_{1}$ and $J_{2}$ are different, that is, when the submarine has not an axial symmetry with respect to the propulsion direction. Specific tracking algorithms for planar and purely angular motions are also considered. The case where $J_{1}=J_{2}$ is discussed in Section 4 . The arguments are very similar to those seen previously and proofs are therefore slightly sketchier. A joint proof of exact controllability is provided in Section 5 .

Finally in Section 6 we show how the tracking procedure can be applied in practice and we illustrate by some examples the features of the proposed approach.

\section{Reminder : continuous dependence of solutions of ordinary dif- ferential equations on parameters}

We recall below a known result about the dependence on the vector field of the solutions of nonautonomous ordinary differential equations.

Proposition 2.1 (Kurzweil and Vorel, 1957) Let $\Omega$ be an open subset of $\mathbf{R}^{m}, m \geq 1$, and $T$ be a positive real number. Denote by $\mathcal{V}$ the set of non-autonomous vector fields defined on $[0, T] \times \Omega$ which are Lebesgue-measurable with respect to $t \in[0, T]$ and smooth with respect to $x \in \Omega$. Consider a sequence $X_{n}$, contained in $\mathcal{V}$, which converges to a vector field $X \in \mathcal{V}$ in the following sense:

$$
\int_{0}^{t} X_{n}(\tau, x) d \tau \rightarrow \int_{0}^{t} X(\tau, x) d \tau \quad \text { as } n \rightarrow \infty,
$$

uniformly with respect to $(t, x) \in[0, T] \times \Omega$. Assume, moreover, that there exists a Lebesgue-integrable function $\psi:[0, T] \rightarrow \mathbf{R}$ such that $\left\|X_{n}(t, x)\right\| \leq \psi(t)$ for every $(t, x) \in[0, T] \times \Omega$ and every $n \in \mathbf{N}$. Then, for every $\epsilon>0$ and every $x_{0} \in \Omega$, there exists $N \in \mathbf{N}$ such that if the Caratheodory solution $x(\cdot)$ of $\dot{x}(t)=X(t, x(t))$ with initial condition $x(0)=x_{0}$ is defined and contained in $\Omega$ on the whole interval $[0, T]$, then for every $n \geq N$ the same is true for the Caratheodory solution $x_{n}(\cdot)$ of $\dot{x}_{n}(t)=X_{n}\left(t, x_{n}(t)\right)$ with the same initial condition $x_{n}(0)=x_{0}$ and, moreover, $\left\|x_{n}(t)-x(t)\right\|<\epsilon$ for every $t \in[0, T]$.

The result stated above (in a more general version which allows for much less regularity of the vector fields with respect to the variable $x$ ) is contained in [13]. Continuity results based on convergence 
of vector fields of the type (5) were first introduced by Giham in [12]. The role of such notion of convergence in control theory is remarkably discussed by Liu and Sussmann in [16]. For a general discussion on Giham's convergence criterion, see [3].

\section{Tracking via backstepping: the case $J_{1} \neq J_{2}$}

This section contains a detailed description of the tracking porcedure under the assumption that $J_{1}$ and $J_{2}$ are different, i.e., that

$$
\gamma=\frac{1}{J_{2}}-\frac{1}{J_{1}} \neq 0
$$

The technical role of such hypothesis becomes evident by fully expanding Kirchhoff's equations, as follows,

$$
\begin{aligned}
\dot{\Pi}_{1} & =\left(\frac{1}{J_{3}}-\frac{1}{J_{2}}\right) \Pi_{2} \Pi_{3}+\mu_{1} P_{2} P_{3}+u_{1}, \\
\dot{\Pi}_{2} & =\left(\frac{1}{J_{1}}-\frac{1}{J_{3}}\right) \Pi_{3} \Pi_{1}+\mu_{2} P_{3} P_{1}+u_{2}, \\
\dot{\Pi}_{3} & =\gamma \Pi_{1} \Pi_{2}+\mu_{3} P_{1} P_{2}, \\
\dot{P}_{1} & =\frac{P_{2} \Pi_{3}}{J_{3}}-\frac{P_{3} \Pi_{2}}{J_{2}} \\
\dot{P}_{2} & =\frac{P_{3} \Pi_{1}}{J_{1}}-\frac{P_{1} \Pi_{3}}{J_{3}} \\
\dot{P}_{3} & =\frac{P_{1} \Pi_{2}}{J_{2}}-\frac{P_{2} \Pi_{1}}{J_{1}}+u_{3},
\end{aligned}
$$

where

$$
\mu_{1}=\frac{1}{M_{3}}-\frac{1}{M_{2}}, \quad \mu_{2}=\frac{1}{M_{1}}-\frac{1}{M_{3}}, \quad \mu_{3}=\frac{1}{M_{2}}-\frac{1}{M_{1}} .
$$

The idea, borrowed from the well-known backstepping procedure, is to look at $\Pi_{1}, \Pi_{2}, P_{3}$, which can be directly tuned by the components of $u$, as control variables in the equations for the remaining three variables $\Pi_{3}, P_{1}, P_{2}$. The structure of such equations and the role played in them by the fictitious control $\left(\Pi_{1}, \Pi_{2}, P_{3}\right)$ are clearly affected by the vanishing of the coefficient $\gamma$.

Lemma 3.1 Assume that $\gamma \neq 0$. Let $T>0$ and fix a smooth curve $(\bar{\Pi}, \bar{P}):[0, T] \rightarrow \mathbf{R}^{6}$. Then there exists a sequence $u^{n}$ contained in $L^{\infty}\left([0, T], \mathbf{R}^{3}\right)$ such that the solutions $\left(\Pi^{n}, P^{n}\right)$ of $(6)-(11)$ corresponding to $u^{n}$ and with initial condition $\left(\Pi^{n}(0), P^{n}(0)\right)=(\bar{\Pi}(0), \bar{P}(0))$ satisfy

$$
\begin{aligned}
\left(\Pi_{1}^{n}(T), \Pi_{2}^{n}(T), P_{3}^{n}(T)\right) & \longrightarrow\left(\bar{\Pi}_{1}(T), \bar{\Pi}_{2}(T), \bar{P}_{3}(T)\right), \\
\left(\Pi_{3}^{n}(t), P_{1}^{n}(t), P_{2}^{n}(t)\right) & \longrightarrow\left(\bar{\Pi}_{3}(t), \bar{P}_{1}(t), \bar{P}_{2}(t)\right), \\
\int_{0}^{t}\left(\Pi_{1}^{n}(\tau), \Pi_{2}^{n}(\tau), P_{3}^{n}(\tau)\right) d \tau & \longrightarrow \int_{0}^{t}\left(\bar{\Pi}_{1}(\tau), \bar{\Pi}_{2}(\tau), \bar{P}_{3}(\tau)\right) d \tau,
\end{aligned}
$$

as $n \rightarrow \infty$, the last two convergences being uniform with respect to $t$ in $[0, T]$. Moreover, there exists $\psi \in L^{1}([0, T], \mathbf{R})$ such that $\left\|\left(\Pi^{n}(t), P^{n}(t)\right)\right\| \leq \psi(t)$ for every $t \in[0, T]$ and every $n \in \mathbf{N}$.

Proof. First notice that the lemma is proved if we can show that its conclusion holds for a (suitable) sequence of smooth curves $t \mapsto\left(\bar{\Pi}^{(n)}(t), \bar{P}^{(n)}(t)\right)$ satisfying

$$
\left(\bar{\Pi}^{(n)}(0), \bar{P}^{(n)}(0)\right)=(\bar{\Pi}(0), \bar{P}(0))
$$


and converging uniformly to $t \mapsto(\bar{\Pi}(t), \bar{P}(t))$. Indeed, such being the case,

$$
\begin{gathered}
\left(\int_{0}^{t} \bar{\Pi}_{1}^{(n)}(\tau) d \tau, \int_{0}^{t} \bar{\Pi}_{2}^{(n)}(\tau) d \tau, \bar{\Pi}_{3}^{(n)}(t), \bar{P}_{1}^{(n)}(t), \bar{P}_{2}^{(n)}(t), \int_{0}^{t} \bar{P}_{3}^{(n)}(\tau) d \tau\right) \\
\longrightarrow\left(\int_{0}^{t} \bar{\Pi}_{1}(\tau) d \tau, \int_{0}^{t} \bar{\Pi}_{2}(\tau) d \tau, \bar{\Pi}_{3}(t), \bar{P}_{1}(t), \bar{P}_{2}(t), \int_{0}^{t} \bar{P}_{3}(\tau) d \tau\right)
\end{gathered}
$$

uniformly with respect to $t \in[0, T]$. The conclusion for $(\bar{\Pi}, \bar{P})$ follows from a simple diagonal procedure. We are therefore free to assume that $(\bar{\Pi}, \bar{P})$ is defined up to a small $\mathcal{C}^{0}$ perturbation which preserves the initial condition.

Since $\Pi_{1}, \Pi_{2}$, and $P_{3}$ are directly tuned by the control, we are justified to write

$$
\begin{aligned}
& \Pi_{1}^{n}(t)=\bar{\Pi}_{1}(t)+v_{1}^{n}(t), \\
& \Pi_{2}^{n}(t)=\bar{\Pi}_{2}(t)+v_{2}^{n}(t), \\
& P_{3}^{n}(t)=\bar{P}_{3}(t)+v_{3}^{n}(t),
\end{aligned}
$$

and to consider $v^{n}=\left(v_{1}^{n}, v_{2}^{n}, v_{3}^{n}\right)$ as control functions. The admissibility of the control functions $u^{n}$ is equivalent to the Lipschitz continuity of $v^{n}$. In what follows, $v^{n}$ will actually be chosen in $\mathcal{C}^{\infty}\left([0, T], \mathbf{R}^{3}\right)$.

We look at $\left(\Pi_{3}^{n}, P_{1}^{n}, P_{2}^{n}\right)$ as at the solution of the Cauchy problem

$$
\frac{d}{d t}\left(\Pi_{3}, P_{1}, P_{2}\right)^{T}=Y\left(t, \Pi_{3}, P_{1}, P_{2}, v^{n}(t)\right), \quad\left(\Pi_{3}(0), P_{1}(0), P_{2}(0)\right)=\left(\bar{\Pi}_{3}(0), \bar{P}_{1}(0), \bar{P}_{2}(0)\right)
$$

where, for every $v=\left(v_{1}, v_{2}, v_{3}\right)$ in $\mathbf{R}^{3}$,

$$
Y\left(t, \Pi_{3}, P_{1}, P_{2}, v\right)=\left(\begin{array}{c}
\gamma\left(\bar{\Pi}_{1}(t)+v_{1}\right)\left(\bar{\Pi}_{2}(t)+v_{2}\right)+\mu_{3} P_{1} P_{2} \\
\frac{P_{2} \Pi_{3}}{J_{3}}-\frac{\left(\bar{P}_{3}(t)+v_{3}\right)\left(\bar{\Pi}_{2}(t)+v_{2}\right)}{J_{2}} \\
\frac{\left(\bar{P}_{3}(t)+v_{3}\right)\left(\bar{\Pi}_{1}(t)+v_{1}\right)}{J_{1}}-\frac{P_{1} \Pi_{3}}{J_{3}}
\end{array}\right) .
$$

Let us rewrite $Y$ in a favorable way. For every $t \in[0, T]$ define

$$
\begin{aligned}
& \bar{\varphi}_{1}(t)=-J_{2} \dot{\bar{P}}_{1}(t)+\frac{J_{2}}{J_{3}} \bar{P}_{2}(t) \bar{\Pi}_{3}(t)-\bar{\Pi}_{2}(t) \bar{P}_{3}(t), \\
& \bar{\varphi}_{2}(t)=J_{1} \dot{\bar{P}}_{2}(t)+\frac{J_{1}}{J_{3}} \bar{P}_{1}(t) \bar{\Pi}_{3}(t)-\bar{\Pi}_{1}(t) \bar{P}_{3}(t), \\
& \bar{\varphi}_{3}(t)=\frac{1}{\gamma} \dot{\bar{\Pi}}_{3}(t)-\frac{\mu_{3}}{\gamma} \bar{P}_{1}(t) \bar{P}_{2}(t)-\bar{\Pi}_{1}(t) \bar{\Pi}_{2}(t) .
\end{aligned}
$$

Then,

$$
Y\left(t, \Pi_{3}, P_{1}, P_{2}, v\right)=\left(\begin{array}{cccc}
\dot{\bar{\Pi}}_{3}+\gamma\left(v_{1} v_{2}-\bar{\varphi}_{3}\right) & +\gamma\left(\bar{\Pi}_{1} v_{2}+\bar{\Pi}_{2} v_{1}\right)+\mu_{3}\left(P_{1} P_{2}-\bar{P}_{1} \bar{P}_{2}\right) \\
\dot{\bar{P}}_{1} & -\frac{1}{J_{2}}\left(v_{2} v_{3}-\bar{\varphi}_{1}\right) & -\frac{1}{J_{2}}\left(\bar{\Pi}_{2} v_{3}+\bar{P}_{3} v_{2}\right) & +\frac{1}{J_{3}}\left(P_{2} \Pi_{3}-\bar{P}_{2} \bar{\Pi}_{3}\right) \\
\dot{\bar{P}}_{2} & +\frac{1}{J_{1}}\left(v_{1} v_{3}-\bar{\varphi}_{2}\right) & +\frac{1}{J_{1}}\left(\bar{\Pi}_{1} v_{3}+\bar{P}_{3} v_{1}\right) & -\frac{1}{J_{3}}\left(P_{1} \Pi_{3}-\bar{P}_{1} \bar{\Pi}_{3}\right)
\end{array}\right) .
$$

The goal is to find a sequence $v^{n}$ in $\mathcal{C}^{\infty}\left([0, T], \mathbf{R}^{3}\right)$ such that $v^{n}(0)=0=v^{n}(T)$,

$$
\int_{0}^{t} v^{n}(\tau) d \tau \longrightarrow 0
$$

uniformly with respect to $t \in[0, T]$, and such that the sequence of solutions $t \mapsto\left(\Pi_{3}^{n}(t), P_{1}^{n}(t), P_{2}^{n}(t)\right)$ of (18) satisfies

$$
\left(\Pi_{3}^{n}(t), P_{1}^{n}(t), P_{2}^{n}(t)\right) \longrightarrow\left(\bar{\Pi}_{3}(t), \bar{P}_{1}(t), \bar{P}_{2}(t)\right)
$$


uniformly with respect to $t \in[0, T]$.

Note that if the triple $\left(\varphi_{1}, \varphi_{2}, \varphi_{3}\right)$ belongs to the set

$$
\mathbf{R}^{3,+}=\left\{(x, y, z) \in \mathbf{R}^{3} \mid x y z>0\right\},
$$

then the system of algebraic equations

$$
\varphi_{1}=v_{2} v_{3}, \quad \varphi_{2}=v_{1} v_{3}, \quad \varphi_{3}=v_{1} v_{2},
$$

has exactly two solutions

$$
v^{ \pm}\left(\varphi_{1}, \varphi_{2}, \varphi_{3}\right)= \pm\left(\sqrt{\frac{\varphi_{2} \varphi_{3}}{\varphi_{1}}}, \sqrt{\frac{\varphi_{1} \varphi_{3}}{\varphi_{2}}}, \sqrt{\frac{\varphi_{1} \varphi_{2}}{\varphi_{3}}}\right) .
$$

Assume for now that the curve described by $\bar{\varphi}=\left(\bar{\varphi}_{1}, \bar{\varphi}_{2}, \bar{\varphi}_{3}\right)$ is contained in $\mathbf{R}^{3,+}$. Let

$$
\bar{v}^{ \pm}(t)=v^{ \pm}(\bar{\varphi}(t)) .
$$

We look for $v^{n}$ in the form

$$
v^{n}(t)=\alpha^{n}(t) \bar{v}^{+}(t)+\left(1-\alpha^{n}(t)\right) \bar{v}^{-}(t),
$$

with $\alpha^{n} \in \mathcal{C}^{\infty}([0, T],[0,1])$. Choose $\alpha^{n}$ in such a way that

$$
\alpha^{n}(0)=\frac{1}{2}=\alpha^{n}(T)
$$

and

$$
\mathcal{L}\left(\left\{t \in[0, T] \mid \alpha^{n}(t) \in(0,1)\right\}\right) \longrightarrow 0
$$

as $n$ tend to infinity, where $\mathcal{L}$ denotes the Lebesgue measure in $\mathbf{R}$. By distributing homogeneously in $[0, T]$ the intervals on which $\alpha^{n}(t)=0$ and those on which $\alpha^{n}(t)=1$, we can, in addition, assume that

$$
\int_{0}^{t}\left(\alpha^{n}(\tau)-\frac{1}{2}\right) d \tau \longrightarrow 0
$$

uniformly with respect to $t \in[0, T]$.

From (29) we immediately deduce (21). The initial and final conditions on $v^{n}$ are ensured by (27), while, according to (23) and (28),

$$
\int_{0}^{t}\left(v_{2}^{n}(\tau) v_{3}^{n}(\tau), v_{1}^{n}(\tau) v_{3}^{n}(\tau), v_{1}^{n}(\tau) v_{2}^{n}(\tau)\right) d \tau \longrightarrow \int_{0}^{t}\left(\bar{\varphi}_{1}(\tau), \bar{\varphi}_{2}(\tau), \bar{\varphi}_{3}(\tau)\right) d \tau
$$

uniformly with respect to $t \in[0, T]$. Therefore,

$$
\int_{0}^{t} Y\left(\tau, \Pi_{3}, P_{1}, P_{2}, v^{n}(\tau)\right) d \tau \longrightarrow \int_{0}^{t} X\left(\tau, \Pi_{3}, P_{1}, P_{2}\right) d \tau
$$

uniformly with respect to $\left(t, \Pi_{3}, P_{1}, P_{2}\right) \in[0, T] \times \Omega$, where $\Omega$ is a compact neighborhood of the target trajectory $\left(\bar{\Pi}_{3}, \bar{P}_{1}, \bar{P}_{2}\right)$ and

$$
X\left(t, \Pi_{3}, P_{1}, P_{2}\right)=\left(\begin{array}{c}
\dot{\bar{\Pi}}_{3}(t)+\mu_{3}\left(P_{1} P_{2}-\bar{P}_{1}(t) \bar{P}_{2}(t)\right) \\
\dot{\bar{P}}_{1}(t)+\frac{1}{J_{3}}\left(P_{2} \Pi_{3}-\bar{P}_{2}(t) \bar{\Pi}_{3}(t)\right) \\
\dot{\bar{P}}_{2}(t)-\frac{1}{J_{3}}\left(P_{1} \Pi_{3}-\bar{P}_{1}(t) \bar{\Pi}_{3}(t)\right)
\end{array}\right) .
$$


Proposition 2.1 implies that $\left(\Pi_{3}^{n}, P_{1}^{n}, P_{2}^{n}\right)$ converges uniformly to the solution of

$$
\frac{d}{d t}\left(\Pi_{3}, P_{1}, P_{2}\right)^{T}=X\left(t, \Pi_{3}, P_{1}, P_{2}\right)
$$

with initial condition $\left(\Pi_{3}(0), P_{1}(0), P_{2}(0)\right)=\left(\bar{\Pi}_{3}(0), \bar{P}_{1}(0), \bar{P}_{2}(0)\right)$. By uniqueness of solutions of regular ordinary differential equations $\left(\Pi_{3}(t), P_{1}(t), P_{2}(t)\right)=\left(\bar{\Pi}_{3}(t), \bar{P}_{1}(t), \bar{P}_{2}(t)\right)$ for every $t \in[0, T]$. This proves the lemma under the hypothesis that the curve $\bar{\varphi}$ is contained in $\mathbf{R}^{3,+}$.

Dropping such assumption requires two fixes: on the one hand, for $\operatorname{arcs}$ of $\bar{\varphi}$ lying in $\mathbf{R}^{3,-}=$ $\{(x, y, z) \mid x y z<0\}$ we consider a suitable convexification procedure; on the other hand, we ensure that the divergence of (24) as $\varphi$ approaches $\mathbf{R}^{3,0}=\mathbf{R}^{3} \backslash\left(\mathbf{R}^{3,+} \cup \mathbf{R}^{3,-}\right)$ does not disrupt the limiting procedure.

With an eye on this second concern, we notice that, up to a $\mathcal{C}^{0}$-small perturbation of $(\bar{\Pi}, \bar{P})$, we can assume that the curve described by $\bar{\varphi}$ satisfies

$$
\dot{\bar{\varphi}}(0) \notin \mathbf{R}^{3,0}
$$

and is in general position with respect to $\mathbf{R}^{3,0}$ on $(0, T]$, i.e., for every $t \in(0, T]$ such that $\bar{\varphi}(t) \in \mathbf{R}^{3,0}$ only one of the coordinates of $\bar{\varphi}(t)$ is equal to zero and $\dot{\bar{\varphi}}(t)$ is transversal to the tangent plane to $\mathbf{R}^{3,0}$ at $\bar{\varphi}(t)$.

Let

$$
I^{\star}=\left\{t \in[0, T] \mid \bar{\varphi}(t) \in \mathbf{R}^{3, \star}\right\}, \quad \star=+,-, 0 .
$$

The assumption on the general position of $\bar{\varphi}$ and (30) guarantee that $I^{0}$ is finite. Equation (25) defines $\bar{v}^{ \pm}$on $I^{+}$. Fix $t_{0} \in I^{0} \backslash\{0\}$ and denote by $j$ the element of $\{1,2,3\}$ such that $\bar{\varphi}_{j}(t)=O\left(\left|t-t_{0}\right|\right)$. Then, for $i \neq j$ there exists $c_{i} \neq 0$ such that $\bar{\varphi}_{i}(t)=c_{i}+O\left(\left|t-t_{0}\right|\right)$. It follows from (24) that

$$
\left\|\bar{v}^{ \pm}(t)\right\|=\frac{c}{\sqrt{\left|t-t_{0}\right|}}+O\left(\sqrt{\left|t-t_{0}\right|}\right), \quad t \in I^{+},
$$

for some $c>0$. It is not hard to check that (31) is satisfied also in the case in which $t_{0}=0$ belongs to $I_{0}$, thanks to $(30)$.

In conclusion, the two maps $\bar{v}^{ \pm}: I^{+} \rightarrow \mathbf{R}^{3}$ are Lebesgue integrable.

Concerning the arcs of $\bar{\varphi}$ contained $n \mathbf{R}^{3,-}$, the idea is to apply the classical relaxation-byconvexification technique to the fictitious control system obtained through the backstepping procedure. Although straightforward in its basic principle (the convex hull of $\mathbf{R}^{3,+}$ is clearly $\mathbf{R}^{3}$ ), the relaxation procedure has some sensitive aspect (e.g., the equi-integrability of $\left(\Pi^{n}, P^{n}\right)$ ). For this reason, and in order to keep the choice of the controls as explicit as possible, we prefer to carry out the procedure in details.

Let, for every $j=1,2,3, D^{j}$ be the 3 -by-3 diagonal matrix $\operatorname{diag}\left(d_{1}, d_{2}, d_{3}\right)$ satisfying $d_{i}=1$ if $i=j$ and $d_{i}=-1$ for $i \neq j$. Let, in addition, $D_{0}$ be the 3-by-3 identity matrix. Notice that, by construction, $D^{j}\left(\mathbf{R}^{3,+}\right)=\mathbf{R}^{3,+}$. Define

$$
\bar{v}^{j, \pm}(t)=D^{j} v^{ \pm}(-\bar{\varphi}(t))
$$

for $t \in I^{-}$and $j=0,1,2,3$. The argument presented above implies that each $\bar{v}^{j, \pm}$ belongs to $L^{1}\left(I^{-}, \mathbf{R}^{3}\right)$.

For every $w=\left(w_{1}, w_{2}, w_{3}\right) \in \mathbf{R}^{3}$ let

$$
\begin{aligned}
|w| & =\left(\left|w_{1}\right|,\left|w_{2}\right|,\left|w_{3}\right|\right), \\
w \oslash w & =\left(w_{2} w_{3}, w_{1} w_{3}, w_{1} w_{2}\right) .
\end{aligned}
$$


An elementary computation shows that

$$
\bar{v}^{j, \pm} \oslash \bar{v}^{j, \pm}=D^{j}|\bar{\varphi}|
$$

For every $t \in I^{-}$the positive cone generated by $D^{0}|\bar{\varphi}(t)|, \ldots, D^{3}|\bar{\varphi}(t)|$ is the whole $\mathbf{R}^{3}$. In particular, there exists a map $\hat{\jmath}: \mathbf{R}^{3,-} \rightarrow\{0,1,2,3\}$ which is constant on each connected component of $\mathbf{R}^{3,-}$ and such that

$$
w=\frac{1}{3}\left(\sum_{j=0}^{3} D^{j}|w|-D^{\hat{\jmath}(w)}|w|\right)
$$

for every $w \in \mathbf{R}^{3,-}$.

We look for $v^{n}$ in the form

$$
v^{n}(t)= \begin{cases}0 & \text { if } \operatorname{dist}\left(t, I^{0}\right)<1 / n, \\ \alpha^{n}(t) \bar{v}^{+}(t)+\left(1-\alpha^{n}(t)\right) \bar{v}^{-}(t) & \text { if } \operatorname{dist}\left(t, I^{0}\right) \geq 1 / n \text { and } t \in I^{+}, \\ \sum_{j=0}^{3} \beta^{j, n}(t)\left(\alpha^{n}(t) \bar{v}^{j,+}(t)+\left(1-\alpha^{n}(t)\right) \bar{v}^{j,-}(t)\right) & \text { if } \operatorname{dist}\left(t, I^{0}\right) \geq 1 / n \text { and } t \in I^{-} .\end{cases}
$$

We choose, as above, the sequence $\alpha^{n}$ in $\mathcal{C}^{\infty}([0, T],[0,1])$ satisfying the initial-and-final condition (27) and the asymptotic conditions (28) and (29). In order to ensure the smoothness of $v^{n}$ on $[0, T]$, we also require that $\alpha^{n}(t)=1 / 2$ if $\operatorname{dist}\left(t, I^{0}\right) \leq 1 / n$. Further asymptotic conditions on the restriction of $\alpha^{n}$ on $I^{-}$will be required below (see (32)).

As for the sequence $\beta^{n}=\left(\beta^{0, n}, \beta^{1, n}, \beta^{2, n}, \beta^{3, n}\right)$, it will be selected in the space $\mathcal{C}^{\infty}\left(I^{-},[0, \sqrt{3}]^{4}\right)$. First of all we require that

$$
\beta^{\hat{\jmath}(\bar{\varphi}(t)), n}(t)=0
$$

for every $t \in I^{-}$and every $n \in \mathbf{N}$, that is, we ask only three components of $\beta^{n}$ to be active on each connected component of $I^{-}$. We assume, moreover, that "most of the time" only one (in turn) of such three components is active and that the three control configurations are homogeneously distributed in time. More precisely, given $0 \leq j \leq 3, t \in[0, T]$, and $n \in \mathbf{N}$, we define

$$
B_{t}^{j, n}=\left\{\tau \in(0, t) \cap I^{-} \mid \beta^{j, n}(\tau)=\sqrt{3}, \beta^{i, n}(\tau)=0 \text { for } i \neq j\right\}
$$

and we ask that

$$
\mathcal{L}\left(B_{t}^{j, n}\right) \longrightarrow \frac{\mathcal{L}\left(\left\{\tau \in(0, t) \cap I^{-} \mid j \neq \hat{\jmath}(\bar{\varphi}(\tau))\right\}\right)}{3}
$$

as $n \rightarrow \infty$, uniformly with respect to $t \in[0, T]$. Moreover, we are free to assume that

$$
\int_{B_{t}^{j, n}}\left(\alpha^{n}(\tau)-\frac{1}{2}\right) d \tau \rightarrow 0
$$

as $n$ tends to infinity, uniformly with respect to $t \in[0, T]$.

A first consequence of the choice of $\alpha^{n}$ and $\beta^{j, n}$ (and in particular of (29) and (32)) is that (21) holds true. In addition,

$$
\int_{0}^{t} v^{n}(\tau) \oslash v^{n}(\tau) d \tau \longrightarrow \int_{0}^{t} \bar{\varphi}(\tau) d \tau
$$

uniformly with respect to $t \in[0, T]$. Upper $L^{1}$ estimates on $\left\|v^{n}(\cdot)\right\|$ and $\left\|v^{n}(\cdot) \oslash v^{n}(\cdot)\right\|$ are given, respectively, by $k_{0}\left\|v^{+}(|\bar{\varphi}(\cdot)|)\right\|$ and $k_{0}\|\bar{\varphi}(\cdot)\|$, for some $k_{0}>0$ large enough.

Therefore, Proposition 2.1 ensures that the sequence $\left(\Pi_{3}^{n}, P_{1}^{n}, P_{2}^{n}\right)$ converges uniformly to the solution $\left(\Pi_{3}(\cdot), P_{1}(\cdot), P_{2}(\cdot)\right)$ of

$$
\frac{d}{d t}\left(\Pi_{3}, P_{1}, P_{2}\right)^{T}=X\left(t, \Pi_{3}, P_{1}, P_{2}\right)
$$


with initial condition $\left(\Pi_{3}(0), P_{1}(0), P_{2}(0)\right)=\left(\bar{\Pi}_{3}(0), \bar{P}_{1}(0), \bar{P}_{2}(0)\right)$. The uniqueness of solutions of regular ordinary differential equations allows us to conclude that

$$
\left(\Pi_{3}(t), P_{1}(t), P_{2}(t)\right)=\left(\bar{\Pi}_{3}(t), \bar{P}_{1}(t), \bar{P}_{2}(t)\right)
$$

for every $t \in[0, T]$.

In order to complete the proof we just need to check that the sequence $\left\|\left(\Pi^{n}(\cdot), P^{n}(\cdot)\right)\right\|$ admits a common $L^{1}$ bound. For what concerns the coordinates $\left(\Pi_{3}, P_{1}, P_{2}\right)$, such bound is guaranteed by the uniform convergence established here above, while for the remaining coordinates, which verify (15)-(17), the bound follows by the $L^{1}$-equiboundedness of the sequence $\left\|v^{n}(\cdot)\right\|$.

Corollary 3.2 If $\gamma \neq 0$ then system $(A, \Pi, r, P)$ is state-trackable.

Proof. Fix a smooth trajectory $(\bar{A}, \bar{r}):[0, T] \rightarrow S O(3) \times \mathbf{R}^{3}$ and an initial condition $\left(\Pi_{0}, P_{0}\right) \in \mathbf{R}^{6}$. Define $\bar{\Pi}, \bar{P}:[0, T] \rightarrow \mathbf{R}^{3}$ through the relations

$$
\bar{\Pi}(t)=J S^{-1}\left(\bar{A}^{-1}(t) \dot{\bar{A}}(t)\right), \quad \bar{P}(t)=M \bar{A}^{-1}(t) \dot{\bar{r}}(t),
$$

and denote by $\left(\Pi^{n}, P^{n}\right)$ the approximating sequence obtained by applying Lemma 3.1 to the trajectory $(\bar{\Pi}, \bar{P})$.

If $(\bar{\Pi}(0), \bar{P}(0))=\left(\Pi_{0}, P_{0}\right)$, then the conclusion follows from Proposition 2.1 applied to the system of equations satisfied by $A$ and $r$ by taking

$$
X_{n}(\tau, A, r)=\left(\begin{array}{c}
A S\left(J^{-1} \Pi^{n}(\tau)\right) \\
A M^{-1} P^{n}(\tau)
\end{array}\right), \quad X(\tau, A, r)=\left(\begin{array}{c}
A S\left(J^{-1} \bar{\Pi}(\tau)\right) \\
A M^{-1} \bar{P}(\tau)
\end{array}\right) .
$$

Let now $(\bar{\Pi}(0), \bar{P}(0))$ be possibly different from $\left(\Pi_{0}, P_{0}\right)$ and fix $\epsilon>0$. Let $\delta>0$ be such that if $0<t<\delta$ then

$$
\|\bar{A}(t)-\bar{A}(0)\|||+\|\bar{r}(t)-\bar{r}(0)\|<\frac{\epsilon}{2} .
$$

As proved above, there exists a control $u \in L^{\infty}\left([\delta, T], \mathbf{R}^{3}\right)$ such that the corresponding trajectory $(A, \Pi, r, P)$ satisfying $(A(\delta), \Pi(\delta), r(\delta), P(\delta))=(\bar{A}(\delta), \bar{\Pi}(\delta), \bar{r}(\delta), \bar{P}(\delta))$ is $\epsilon / 3$-close to the target trajectory in the coordinates $(A, r)$ on the interval $[\delta, T]$.

Fixed $u(\cdot)$, we can chose $\eta>0$ such that the trajectory corresponding to $u(\cdot)$ and with initial condition in a $\eta$-neighborhood of $(\bar{A}(\delta), \bar{\Pi}(\delta), \bar{r}(\delta), \bar{P}(\delta))$ is $\epsilon / 2$-close to the target trajectory in the coordinates $(A, r)$.

Fix now a smooth trajectory $(\hat{A}, \hat{r}):[0, \delta] \rightarrow S O(3) \times \mathbf{R}^{3}$ such that $(\hat{A}(0), \hat{r}(0))=(\bar{A}(0), \bar{r}(0))$,

$$
\|\hat{A}(t)-\bar{A}(0) \mid\|+\|\hat{r}(t)-\bar{r}(0)\|<\frac{\epsilon}{2} \quad \text { for every } t \in[0, \delta],
$$

and $(\hat{\Pi}(0), \hat{P}(0))=\left(\Pi_{0}, P_{0}\right),(\hat{\Pi}(\delta), \hat{P}(\delta))=(\bar{\Pi}(\delta), \bar{P}(\delta))$, where $(\hat{\Pi}, \hat{P})$ are obtained from $(\hat{A}, \hat{r})$ following (33).

Apply Lemma 3.1 in order to obtain a control $v \in L^{\infty}\left([0, \delta], \mathbf{R}^{3}\right)$ whose corresponding trajectory having $(\hat{A}(0), \hat{r}(0))$ as initial condition $\min \{\epsilon / 2, \eta\}$-approximates $(\hat{A}, \hat{r})$. Then the concatenation of $v$ and $u$ provides a control such that the corresponding trajectory starting from $(\hat{A}(0), \hat{r}(0))$ is an $\epsilon$-approximation of $(\hat{A}, \hat{r})$ on $[0, T]$.

Remark 3.3 The results stated in Lemma 3.1 include the approximate controllability of Kirchhoff's equations, i.e., of system $(\Pi, P)$. An elementary computation shows, moreover, that the family of vector fields defining such control system is Lie bracket generating (see [2, Lemma 1]). It follows from Krener theorem (see, for instance, [1, Corollary 8.3]) that system $(\Pi, P)$ is completely controllable in arbitrary small time and, therefore, exactly controllable. 
A non-secondary asset of the construction lying behind Lemma 3.1 is its generality, since an approximating sequence is obtained almost algorithmically from the target trajectory. (The only algorithmically implicit point, in which the procedure can depend on the target, is the eventual choice of an approximating curve $\left(\Pi^{\prime}, P^{\prime}\right)$ rendering $\bar{\varphi}$ transversal to $\mathbf{R}^{3,0}$.) However, in some special cases a more adapted approach looks preferable, giving rise to more natural results.

\subsection{First special case: planar motion}

Consider the case in which the control $u_{1}$ is not active, i.e.,

$$
u_{1} \equiv 0,
$$

and the submarine at time $t=0$ satisfies $\Pi_{1}(0)=\Pi_{3}(0)=P_{2}(0)=0$. The unique solution of Kirchhoff's Cauchy problem satisfies

$$
\Pi_{1} \equiv \Pi_{3} \equiv P_{2} \equiv 0 .
$$

The corresponding trajectory $t \mapsto r(t)$ is therefore constrained on a plane. (A perfectly symmetric situation is the one in which $u_{2}, \Pi_{2}, \Pi_{3}$ and $P_{1}$ are identically equal to zero.)

The case in which the target trajectory is planar and the initial condition on the momenta satisfies (35) can be treated, instead of using the general procedure described in the previous section, as follows: we freeze the control $u_{1}$, i.e., we impose (34) to hold on $[0, T]$, and we deal with the simplified Kirchhoff equations

$$
\begin{aligned}
\dot{\Pi}_{2} & =\mu_{2} P_{1} P_{3}+u_{2}, \\
\dot{P}_{1} & =-\frac{P_{3} \Pi_{2}}{J_{2}}, \\
\dot{P}_{3} & =\frac{P_{1} \Pi_{2}}{J_{2}}+u_{3} .
\end{aligned}
$$

Using the same notations as in the proof of Lemma 3.1, it turns out that $\bar{\varphi}_{2} \equiv \bar{\varphi}_{3} \equiv 0$ and the algebraic system of equations (23) simplifies to

$$
\varphi_{1}=v_{2} v_{3} .
$$

The same backstepping approach introduced in the previous section proves the state-trackability of planar motions by approximating trajectories which are themselves planar. Many technical difficulties in the proof, moreover, get simpler. Notice that, since the curve $\bar{\varphi}$ lies in $\mathbf{R}^{3,0}$ the general non-planar construction of Lemma 3.1 would give rise to a much more complicated approximating strategy.

\subsection{Second special case: purely rotational motion}

The second special case we consider is the one where $P \equiv 0$, i.e., $r$ is constant and only the control $\mathcal{T}$ is active. Under such restriction the control system $(A, \Pi, r, P)$ becomes

$$
\begin{aligned}
& \dot{A}=A S\left(J^{-1} \Pi\right), \\
& \dot{\Pi}=\Pi \times \omega+\mathcal{T},
\end{aligned}
$$


which can be written explicitly as

$$
\begin{aligned}
\dot{A} & =A S\left(J^{-1} \Pi\right) \\
\dot{\Pi}_{1} & =\left(\frac{1}{J_{3}}-\frac{1}{J_{2}}\right) \Pi_{2} \Pi_{3}+u_{1}, \\
\dot{\Pi}_{2} & =\left(\frac{1}{J_{1}}-\frac{1}{J_{3}}\right) \Pi_{3} \Pi_{1}+u_{2}, \\
\dot{\Pi}_{3} & =\gamma \Pi_{1} \Pi_{2} .
\end{aligned}
$$

We will refer to such control system as to "system $(A, \Pi)$ ".

System $(A, \Pi)$ is clearly non-controllable when $\gamma=0$. We prove below that, somehow conversely, the assumption $\gamma \neq 0$ is sufficient to guarantee that system $(A, \Pi)$ is state-trackable and exactly controllable.

Proposition 3.4 Let $\gamma \neq 0$. Fix $T>0, \Pi_{0} \in \mathbf{R}^{3}$ and a smooth trajectory $\bar{A}:[0, T] \rightarrow S O(3)$. Then for every $\epsilon>0$ there exists a measurable bounded control $u=\left(u_{1}, u_{2}\right):[0, T] \rightarrow \mathbf{R}^{2}$ such that the corresponding solution $(A(\cdot), \Pi(\cdot))$ of system $(A, \Pi)$ with initial condition $(A(0), \Pi(0))=\left(\bar{A}(0), \Pi_{0}\right)$ verifies, for every time $t$ in $[0, T],\||\bar{A}(t)-A(t)|\|<\epsilon$. Moreover, $u$ can be chosen in such a way that

$$
\left\|\Pi(T)-J S^{-1}\left(\bar{A}^{-1}(T) \dot{\bar{A}}(T)\right)\right\| \leq \epsilon .
$$

We skip the proof of Proposition 3.4, since it follows faithfully what done for Lemma 3.1 and Proposition 3.2. (Actually, since the ratio between controls and coordinates is higher for system $(A, \Pi)$ than for system $(A, \Pi, r, P)$, many technical difficulties are simplified or disappear altogether.)

Proposition 3.5 Let $\gamma \neq 0$. Then system $(A, \Pi)$ is exactly controllable.

Proof. A consequence of Proposition 3.4 is that system $(A, \Pi)$ is approximatively controllable in arbitrary short time. Therefore, if we prove that the family of vector fields defining system $(A, \Pi)$ satisfies the Lie bracket generating condition then it follows that system $(A, \Pi)$ is short-time completly controllabile (and thus exactly controllable, due to the presence of the equilibrium $(\operatorname{Id}, 0)$ ).

The Lie bracket computation can be found in a paper by Crouch ([10]), where the author proves a basically equivalent result to Proposition 3.5 , namely the complete controllability of system $(A, \Pi)$ in the case $\gamma \neq 0$ ( $T$ free), under the assumption that the set of admissible controls is a bounded rectangle.

Remark 3.6 Controllability in the case $\gamma=0$ can be treated similarly, although aiming at a weaker result (which will be used in the following). Assuming that $\gamma=0$ and fixing $\bar{\Pi}_{3} \in \mathbf{R}$, we can prove that system $(A, \Pi)$ restricted to $S O(3) \times \mathbf{R}^{2} \times\left\{\bar{\Pi}_{3}\right\}$ is (well defined and) exactly controllable. Indeed, Crouch's computations and reasonings (in particular, the application of a general result by Bonnard on controllability of control systems with Poisson stable drift [6]) show that any such restricted system is completely controllable with bounded controls. Due to the special structure of the system, however, the set of admissible trajectories (corresponding to some $u=\left(u_{1}, u_{2}\right) \in L^{\infty}\left([0, T], \mathbf{R}^{2}\right)$ ) is invariant by time-rescaling.

\section{State-trackability: the case $\gamma=0$}

In this section we complete the proof of Theorem 1.4. According to Proposition 3.4, we are left to tackle the case where $\gamma=0$ and $\mu_{3} \neq 0$. We start from the counterpart of Lemma 3.1. Notice that (12)-(13) are replaced here by the weaker asymptotic relations (42)-(43). 
Lemma 4.1 Assume that $\gamma=0$ and $\mu_{3} \neq 0$. Let $T>0$ and fix a smooth curve $(\bar{\Pi}, \bar{P}):[0, T] \rightarrow \mathbf{R}^{6}$. Then there exists a sequence $u^{n}$ contained in $L^{\infty}\left([0, T], \mathbf{R}^{3}\right)$ such that the sequence $\left(\Pi^{n}, P^{n}\right)$ of solutions of (6)-(11) corresponding to $u^{n}$ and with initial condition $\left(\Pi^{n}(0), P^{n}(0)\right)=(\bar{\Pi}(0), \bar{P}(0))$ satisfies

$$
\begin{aligned}
\left(\Pi_{1}^{n}(T), \Pi_{2}^{n}(T), P_{1}^{n}(T), P_{2}^{n}(T), P_{3}^{n}(T)\right) & \rightarrow\left(\bar{\Pi}_{1}(T), \bar{\Pi}_{2}(T), \bar{P}_{1}(T), \bar{P}_{2}(T), \bar{P}_{3}(T)\right), \\
\Pi_{3}^{n}(t) & \rightarrow \bar{\Pi}_{3}(t), \\
\int_{0}^{t}\left(\Pi_{1}^{n}(\tau), \Pi_{2}^{n}(\tau), P_{1}^{n}(\tau), P_{2}^{n}(\tau), P_{3}^{n}(\tau)\right) d \tau & \rightarrow \int_{0}^{t}\left(\bar{\Pi}_{1}(\tau), \bar{\Pi}_{2}(\tau), \bar{P}_{1}(\tau), \bar{P}_{2}(\tau), \bar{P}_{3}(\tau)\right) d \tau
\end{aligned}
$$

as $n \rightarrow \infty$, the last two convergences being uniform with respect to $t$ in $[0, T]$.

Proof. The idea, detailed below, is to apply twice the backstepping procedure developed in the proof of Lemma 3.1: first, by interpreting $P_{1}$ and $P_{2}$ as control variables in the equation for $\Pi_{3}$, we single out a sequence $\left(\hat{\Pi}_{3}^{n}, \hat{P}_{1}^{n}, \hat{P}_{2}^{n}\right)$ such that $\hat{\Pi}_{3}^{n}$ converges uniformly to $\bar{\Pi}_{3}$. In a second step each element of the sequence is approximated uniformly using the three variables $\left(\Pi_{1}, \Pi_{2}, P_{3}\right)$ as controls.

Fix a sequence of smooth curves $w^{n}=\left(w_{1}^{n}, w_{2}^{n}\right):[0, T] \rightarrow \mathbf{R}^{2}$ such that

$$
w^{n}(0)=0=w^{n}(T)
$$

and

$$
\begin{aligned}
\int_{0}^{t} w^{n}(\tau) d \tau & \longrightarrow 0 \\
\int_{0}^{t} w_{1}^{n}(\tau) w_{2}^{n}(\tau) d \tau & \longrightarrow \frac{\bar{\Pi}_{3}(t)-\bar{\Pi}_{3}(0)}{\mu_{3}}-\int_{0}^{t} \bar{P}_{1}(\tau) \bar{P}_{2}(\tau) d \tau,
\end{aligned}
$$

uniformly with respect to $t \in[0, T]$. Condition (47) can be guaranteed by imposing that $w^{n}$ satisfies

$$
w_{1}^{n}(t) w_{2}^{n}(t)=\left(\dot{\bar{\Pi}}_{3}(t) / \mu_{3}\right)-\bar{P}_{1}(t) \bar{P}_{2}(t)
$$

on a subset of $[0, T]$ which converges to $[0, T]$ in measure as $n$ tends to infinity. The asymptotic condition (46) can be ensured by fast oscillating between opposite-sign solutions of (48).

For every $n \in \mathbf{N}$ define

$$
\begin{aligned}
\hat{P}_{1}^{n}(t) & =\bar{P}_{1}(t)+w_{1}^{n}(t) \\
\hat{P}_{2}^{n}(t) & =\bar{P}_{2}(t)+w_{2}^{n}(t) \\
\hat{\Pi}_{3}^{n}(t) & =\bar{\Pi}_{3}(0)+\mu_{3} \int_{0}^{t} \hat{P}_{1}^{n}(\tau) \hat{P}_{2}^{n}(\tau) d \tau .
\end{aligned}
$$

The choice made on $w^{n}$ implies that $\hat{\Pi}_{3}^{n}$ converges uniformly to $\bar{\Pi}_{3}$.

For every $n \in \mathbf{N}$ we look for a sequence $v^{n, k}=\left(v_{1}^{n, k}, v_{2}^{n, k}, v_{3}^{n, k}\right)$ in $\mathcal{C}^{\infty}\left([0, T], \mathbf{R}^{3}\right)$ such that

$$
v^{n, k}(0)=0=v^{n, k}(T)
$$

and

$$
\begin{aligned}
\int_{0}^{t} v^{n, k}(\tau) d \tau & \longrightarrow 0 \\
\left(\Pi_{3}^{n, k}(t), P_{1}^{n, k}(t), P_{2}^{n, k}(t)\right) & \longrightarrow\left(\hat{\Pi}_{3}^{n}(t), \hat{P}_{1}^{n}(t), \hat{P}_{2}^{n}(t)\right)
\end{aligned}
$$


as $k$ tends to infinity, uniformly with respect to $t \in[0, T]$, where $t \mapsto\left(\Pi_{3}^{n, k}(t), P_{1}^{n, k}(t), P_{2}^{n, k}(t)\right)$ is the solution of

$$
\frac{d}{d t}\left(\Pi_{3}, P_{1}, P_{2}\right)^{T}=Y\left(t, \Pi_{3}, P_{1}, P_{2}, v^{n, k}(t)\right), \quad\left(\Pi_{3}(0), P_{1}(0), P_{2}(0)\right)=\left(\bar{\Pi}_{3}(0), \bar{P}_{1}(0), \bar{P}_{2}(0)\right)
$$

with $Y$ defined as in (19).

This can be done by defining

$$
\begin{aligned}
& \hat{\varphi}_{1}^{n}(t)=-J_{2} \dot{\hat{P}}_{1}^{n}(t)+\frac{J_{2}}{J_{3}} \hat{P}_{2}^{n}(t) \hat{\Pi}_{3}^{n}(t)-\bar{P}_{3}(t) \bar{\Pi}_{2}(t), \\
& \hat{\varphi}_{2}^{n}(t)=J_{1} \dot{\hat{P}}_{2}^{n}(t)+\frac{J_{1}}{J_{3}} \hat{P}_{1}^{n}(t) \hat{\Pi}_{3}^{n}(t)-\bar{P}_{3}(t) \bar{\Pi}_{1}(t),
\end{aligned}
$$

and by selecting $v^{n, k}$ such that (49) and (50) hold, and, in addition,

$$
\begin{gathered}
\int_{0}^{t}\left(\hat{\varphi}_{1}^{n}(\tau)-v_{2}^{n, k}(\tau) v_{3}^{n, k}(\tau)\right) d \tau \quad \longrightarrow 0 \\
\int_{0}^{t}\left(\hat{\varphi}_{2}^{n}(\tau)-v_{1}^{n, k}(\tau) v_{3}^{n, k}(\tau)\right) d \tau \quad \longrightarrow 0
\end{gathered}
$$

uniformly with respect to $t \in[0, T]$. Then, as $k$ tends to infinity, $\left(\Pi_{3}^{n, k}(t), P_{1}^{n, k}(t), P_{2}^{n, k}(t)\right)$ tends uniformly with respect to $t \in[0, T]$ to the solution of

$$
\left\{\begin{array}{l}
\dot{\Pi}_{3}=\mu_{3} P_{1} P_{2}, \\
\dot{P}_{1}=\dot{\hat{P}}_{1}^{n}+\frac{1}{J_{3}}\left(P_{2} \Pi_{3}-\hat{P}_{2}^{n} \hat{\Pi}_{3}^{n}\right), \\
\dot{P}_{2}=\dot{\hat{P}}_{2}^{n}-\frac{1}{J_{3}}\left(P_{1} \Pi_{3}-\hat{P}_{1}^{n} \hat{\Pi}_{3}^{n}\right),
\end{array} \quad\left(\Pi_{3}(0), P_{1}(0), P_{2}(0)\right)=\left(\bar{\Pi}_{3}(0), \bar{P}_{1}(0), \bar{P}_{2}(0)\right),\right.
$$

i.e., to $\left(\hat{\Pi}_{3}^{n}(t), \hat{P}_{1}^{n}(t), \hat{P}_{2}^{n}(t)\right)$. The conclusion follows from a diagonal argument.

The same proof as the one of Corollary 3.2 shows how Lemma 4.1 leads to the state-trackability of system $(A, \Pi, r, P)$ in the case where $\gamma=0$ and $\mu_{3} \neq 0$.

\section{$5 \quad$ Exact controllability of system $(A, \Pi, r, P)$}

The proof of the exact controllability of system $(A, \Pi, r, P)$ lacks a last ingredient, which is provided by the following lemma.

Lemma 5.1 System $(\Pi, P)$ is exactly controllable when $\gamma=0$ and $\mu_{3} \neq 0$.

Proof. Let $\gamma=0$ and $\mu_{3} \neq 0$. Lemma 4.1 guarantees approximate short-time controllability for system $(\Pi, P)$. As noticed in Remark 3.3, exact controllability follows if we prove that the system is Lie bracket generating.

Let $X_{0}, \ldots, X_{3}$ be the four vector fields on $\mathbf{R}^{6}$ such that system $(\Pi, P)$ is given by

$$
(\dot{\Pi}, \dot{P})=X_{0}(\Pi, P)+\sum_{i=1}^{3} u_{i} X_{i}(\Pi, P) \text {. }
$$

Then a computation shows that

$$
\begin{aligned}
{\left[X_{1},\left[X_{0}, X_{3}\right]\right](\Pi, P) } & =\left(0,0,0,0,-\frac{1}{J_{1}}, 0\right)^{T}, \\
{\left[X_{2},\left[X_{0}, X_{3}\right]\right](\Pi, P) } & =\left(0,0,0, \frac{1}{J_{1}}, 0,0\right)^{T}, \\
X^{*}=\left[\left[X_{1},\left[X_{0}, X_{3}\right]\right],\left[X_{3},\left[X_{0},\left[X_{0}, X_{2}\right]\right]\right]\right](\Pi, P) & =\left(0,0,-\frac{\mu_{3}}{J_{1}^{2}}, 0,0,0\right)^{T},
\end{aligned}
$$


and thus the six (constant) vector fields $X_{1}, X_{2}, X_{3},\left[X_{1},\left[X_{0}, X_{3}\right]\right],\left[X_{2},\left[X_{0}, X_{3}\right]\right]$, and $X^{*}$ are everywhere linearly independent.

Proof of Theorem 1.3. Fix an initial and a final condition $\left(A_{0}, \Pi_{0}, r_{0}, P_{0}\right)$ and $\left(A_{f}, \Pi_{f}, r_{f}, P_{f}\right)$ for system $(A, \Pi, r, P)$. Fix, in addition, a positive time $T$.

From the exact controllability of Kirchhoff's equations (see Remark 3.3 and Lemma 5.1) it follows that there exist $\left(A_{0}^{\prime}, r_{0}^{\prime}\right),\left(A_{f}^{\prime}, r_{f}^{\prime}\right)$ in $S O(3) \times \mathbf{R}^{3}$ and two admissible controls defined on an interval of length $T / 5$, the first one steering system $(A, \Pi, r, P)$ from $\left(A_{0}, \Pi_{0}, r_{0}, P_{0}\right)$ to $\left(A_{0}^{\prime}, 0, r_{0}^{\prime}, 0\right)$ and the second one from $\left(A_{f}^{\prime}, 0, r_{f}^{\prime}, 0\right)$ to $\left(A_{f}, \Pi_{f}, r_{f}, P_{f}\right)$.

Proposition 3.5 and Remark 3.6, moreover, imply that, for every $A_{0}^{\prime \prime}, A_{f}^{\prime \prime} \in S O(3)$, system $(A, \Pi, r, P)$ can be driven in time $T / 5$ from $\left(A_{0}^{\prime}, 0, r_{0}^{\prime}, 0\right)$ to $\left(A_{0}^{\prime \prime}, 0, r_{0}^{\prime}, 0\right)$ and from $\left(A_{f}^{\prime \prime}, 0, r_{f}^{\prime}, 0\right)$ to $\left(A_{f}^{\prime}, 0, r_{f}^{\prime}, 0\right)$.

It is now physically intuitive how to choose $A_{0}^{\prime \prime}$ and $A_{f}^{\prime \prime}$ in order to guarantee the existence of a control steering system $(A, \Pi, r, P)$ from $\left(A_{0}^{\prime \prime}, 0, r_{0}^{\prime}, 0\right)$ to $\left(A_{f}^{\prime \prime}, 0, r_{f}^{\prime}, 0\right)$ in time $T / 5$ : we take $A_{0}^{\prime \prime}=A_{f}^{\prime \prime}$ to be any rotation such that $r_{f}^{\prime}-r_{0}^{\prime}$ belongs to the axis spanned by $A_{0}^{\prime \prime} e_{3}$, with $e_{3}=(0,0,1)^{T}$. With this choice, and taking $\mathcal{T} \equiv 0$, system $(A, \Pi, r, P)$ is nothing else that a double integrator on the two-dimensional space $A \equiv A_{0}^{\prime \prime}, \Pi \equiv 0, r, v \in \operatorname{span}\left(A_{0}^{\prime \prime} e_{3}\right)$.

It is well-known that such system is exactly controllable and this concludes the proof of Theorem 1.3 .

\section{Numerical simulations}

The algorithm presented in section 3 has been implemented with Scilab. For our example, we have chosen the following constants:

$$
\begin{array}{lll}
J_{1}=1 & J_{2}=2 & J_{3}=3 \\
M_{1}=1 & M_{2}=2 & M_{3}=3 .
\end{array}
$$

The trajectory to be tracked is given by

$$
\begin{array}{ll}
\bar{\Pi}_{1}: t \mapsto 1 & 1+t \\
\bar{\Pi}_{2}: t \mapsto \sin (t) \\
\bar{\Pi}_{3}: t \mapsto \cos (t) \\
\bar{P}_{1}: t \mapsto 1+t^{2} \\
\bar{P}_{2}: t \mapsto 1+t \\
\bar{P}_{3}: t \mapsto 1-t
\end{array}
$$

At time $t=0$, the body is such that $\Pi_{1}=\Pi_{3}=P_{1}=P_{2}=P_{3}=1$ and $\Pi_{2}=0$, that is to say the initial conditions are the same for the actual trajectory of the immersed body and the tracked trajectory.

The implementation of the algorithm presented in Section 3 is done in the open loop way, that is we do not use feedback. This way of implementation is known to be unstable but is a good way to test the stability of the method. Implementation is done using the standard Scilab routines (in particular the ode solver of ODE's). The time interval between two evaluations is forced to be less than $10^{-5}$. Equations are solved for time $t$ between 0 and 3, that is we do at least $3.10^{5}$ evaluations of the positions, resolutions of equations (23) and computation of the corresponding controls. The total computation time is less than five minutes using a standard office desktop.

On the graphs corresponding to the directly tuned coordinates $P_{3}, \Pi_{1}$, and $\Pi_{2}$ (see Figures 3,4 , 5 ), we have represented in blue the evolution of $P_{3}, \Pi_{1}$, and $\Pi_{2}$ respectively. On the same graphs, the evolution of the tracked trajectory $\bar{P}_{3}, \bar{\Pi}_{1}$, and $\bar{\Pi}_{2}$ are represented in black. As expected, the tracked trajectory is much smoother than the actual one but, as desired, the averages of the two 


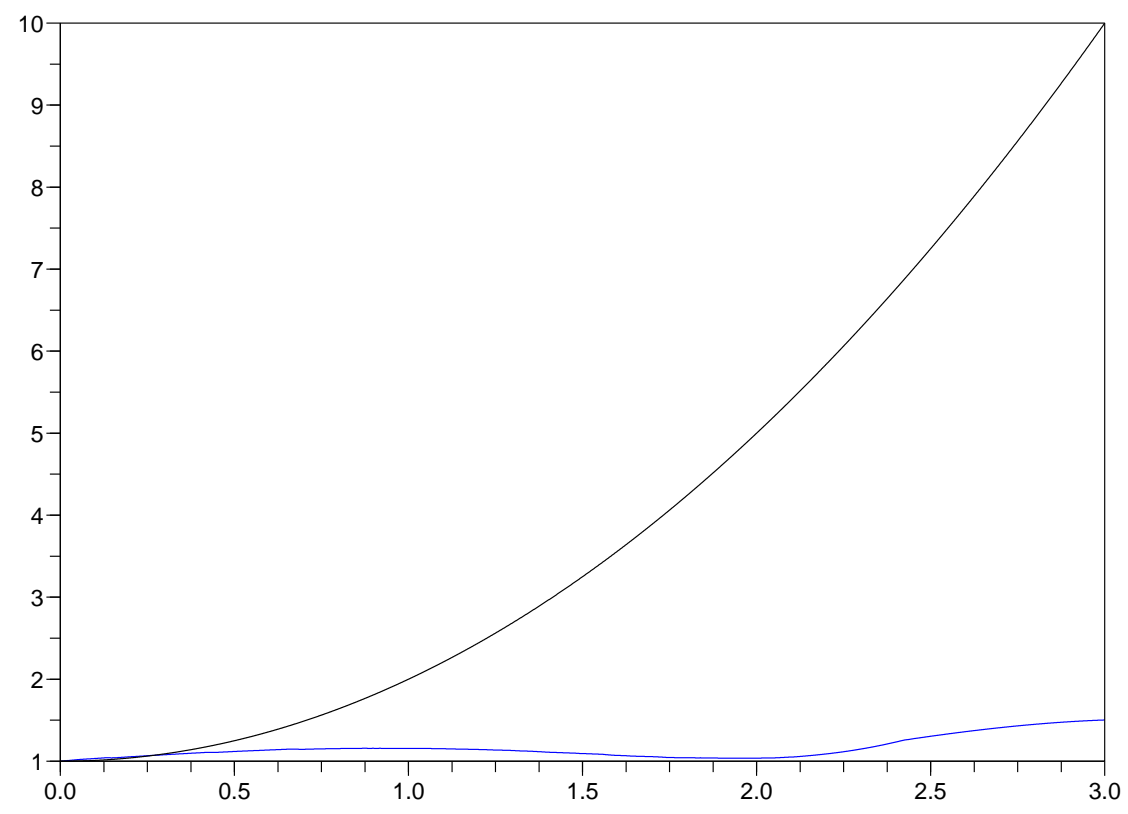

Figure 1: Evolution of the $P_{1}$ coordinate with respect to time

trajectories on small time intervals remain the same for the tracked and the tracking trajectory, as requested by (14). On the graphs corresponding to the indirectly tuned coordinates $P_{1}, P_{2}$ and $\Pi_{3}$ (see Figures 1, 2, and 6) one sees that for small time (say $0 \leq t \leq 0.3$ ), the tracking trajectories remain very close to the corresponding tracked trajectories. After a while (say for $t \geq 0.3$ ) the error cumulation is too large to allow good tracking. However, in view of the good initial behaviour of the control system, one may hope that a suitable feedback procedure will allow precise large time tracking. Such a feedback procedure is still under investigation.

\section{References}

[1] A. A. Agrachev and Yu. L. Sachkov, Control Theory from the Geometric Viewpoint, Springer-Verlag, Berlin, 2004.

[2] A. Astolfi, D. Chhabra, and R. Ortega, Asymptotic stabilization of some equilibria of an underactuated underwater vehicle, Systems and Control Letters, 45 (2002), pp. 193-206.

[3] D. C. Biles, Continuous dependence of nonmonotonic discontinuous differential equations, Trans. Amer. Math. Soc., 339 (1993), pp. 507-524.

[4] A. M. Bloch, P. S. Krishnaprasad, J. E. Marsden, and G. Sanchez de Alvarez, Stabilization of rigid body dynamics by internal and external torques, Automatica, 28 (1992), pp. $745-756$.

[5] A. M. Bloch, N. E. Leonard, and J. E. Marsden, Stabilization of mechanical systems using controlled Lagrangians, Proceedings of IEEE Conf. Decision and Control, San Diego, CA, pp. 2356-2361, 1997. 


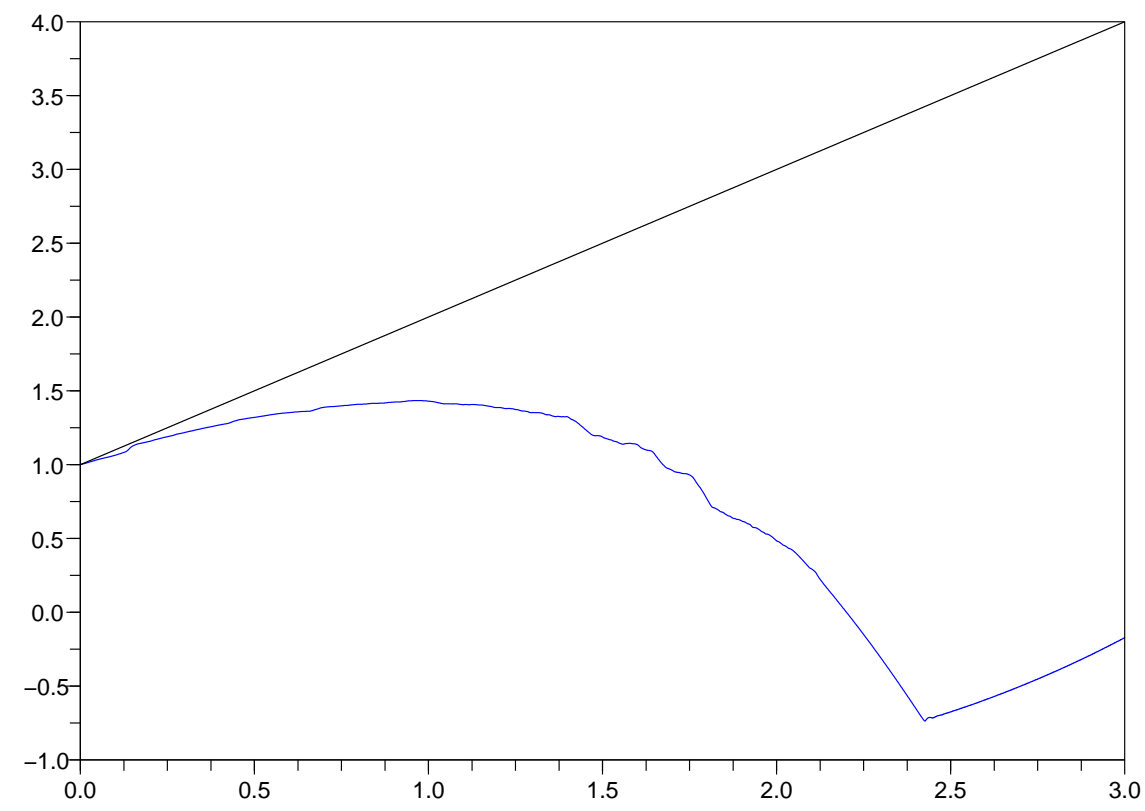

Figure 2: Evolution of the $P_{2}$ coordinate with respect to time

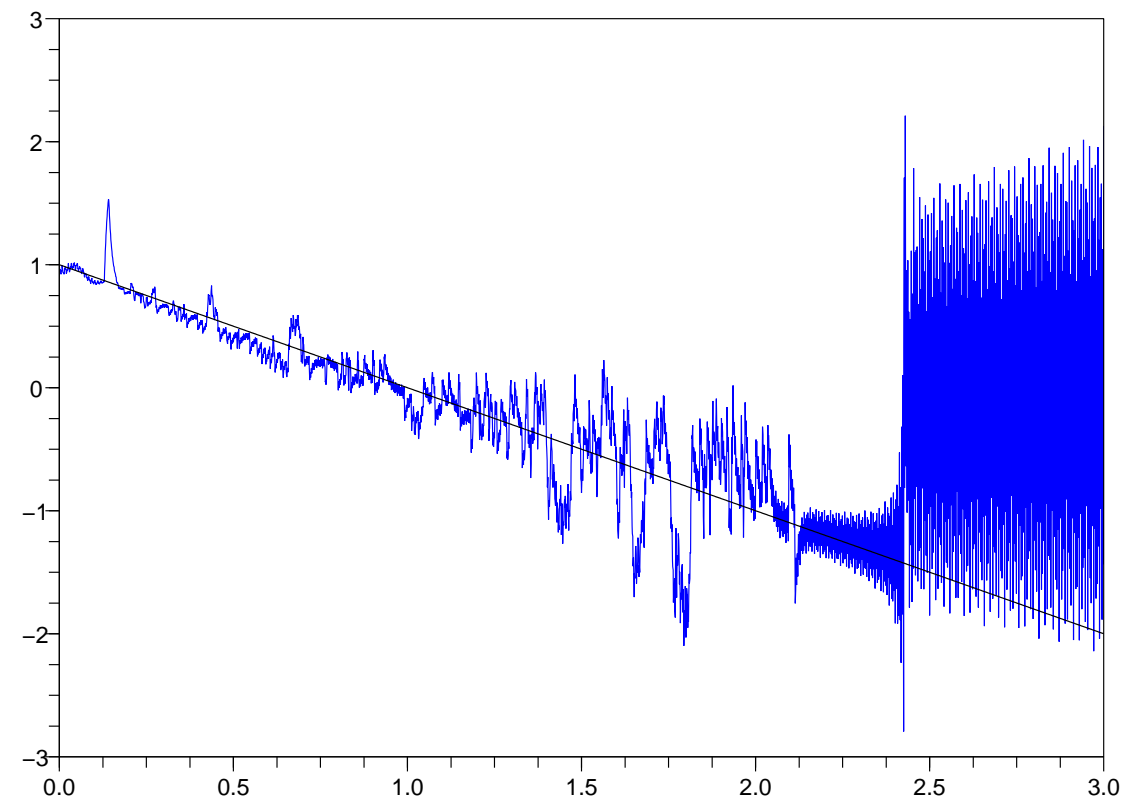

Figure 3: Evolution of the $P_{3}$ coordinate with respect to time 


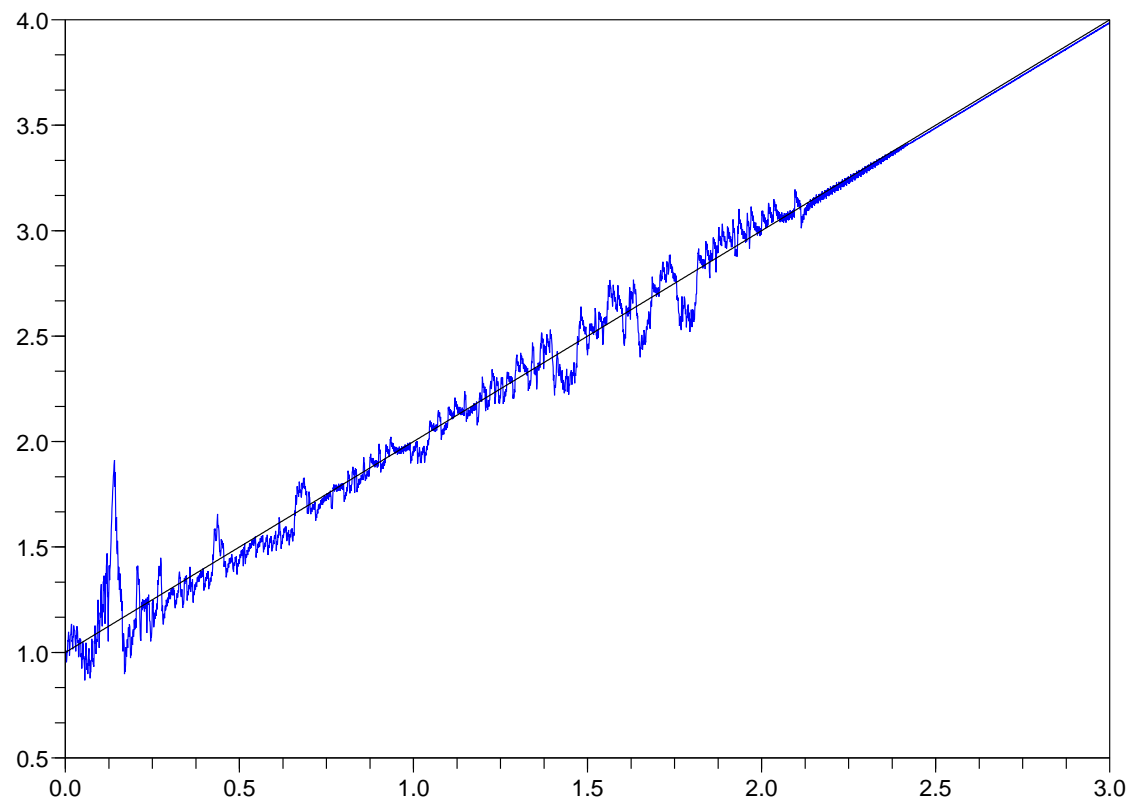

Figure 4: Evolution of the $\Pi_{1}$ coordinate with respect to time

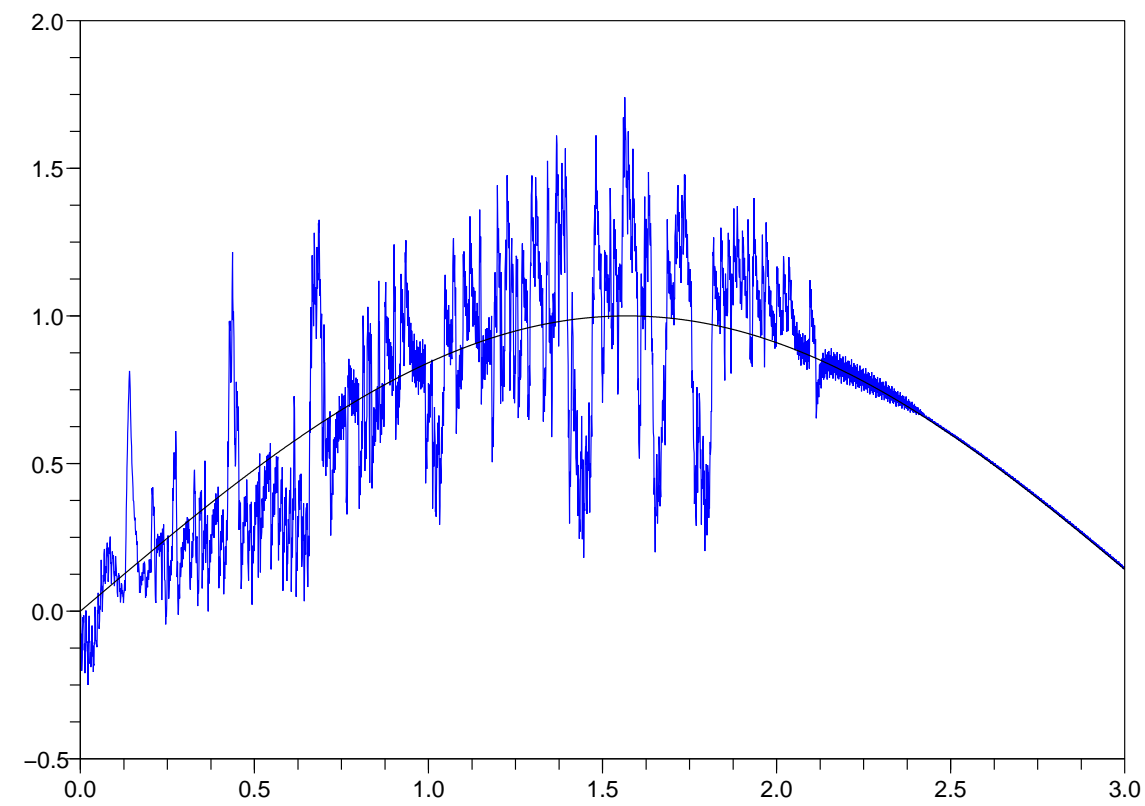

Figure 5: Evolution of the $\Pi_{2}$ coordinate with respect to time 


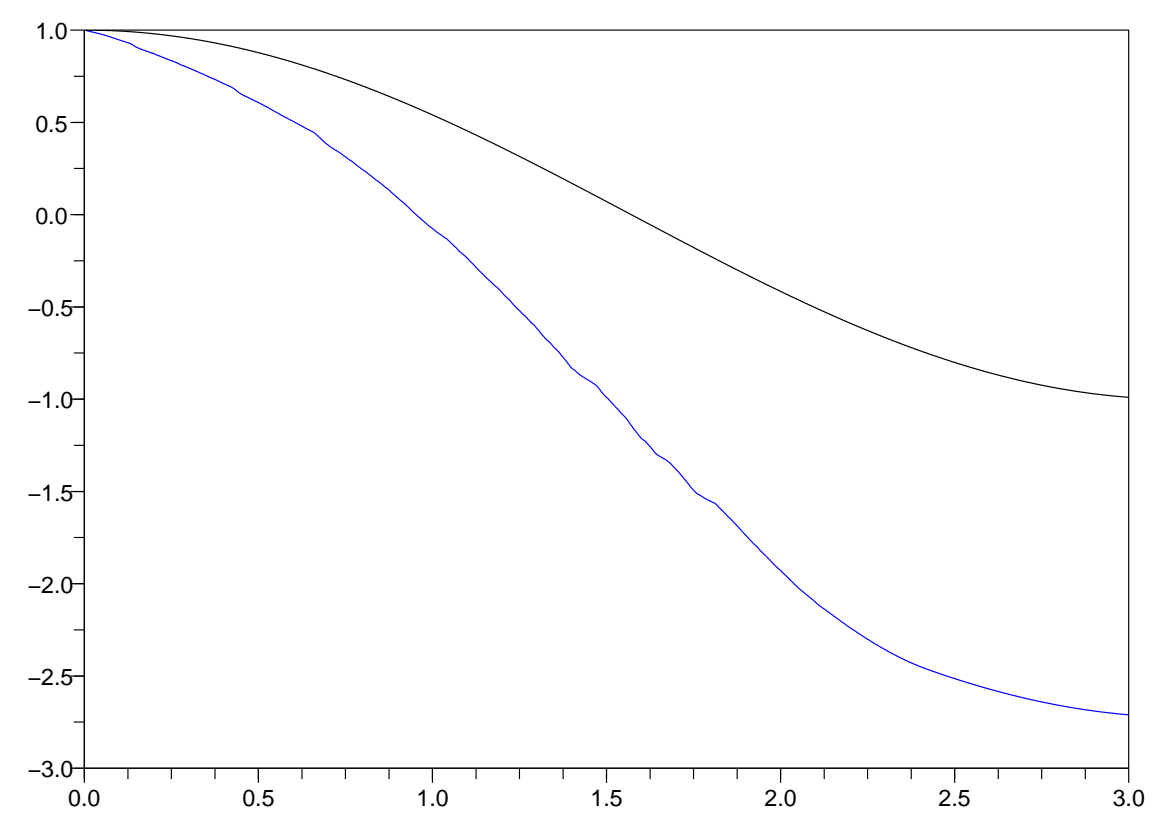

Figure 6: Evolution of the $\Pi_{3}$ coordinate with respect to time

[6] B. Bonnard, Contrôlabilité de systèmes mécaniques sur les groupes de Lie (French), J. Control Optim., 22 (1984), pp. 711-722.

[7] F. Bullo and N. E. Leonard, Motion Control for Underactuated Mechanical Systems on Lie Groups, Proceedings of the European Control Conference, Brussels, Belgium, pp. 480-485, 1997.

[8] M. Chyba, H. Maurer, H. J. Sussmann, and G. Vossen, Underwater vehicles: The minimum time problem, Proceedings of IEEE Conf. Decision and Control, Bahamas, pp. 1370-1375, 2004 .

[9] M. Chyba, N. E. Leonard, and E. D. Sontag, Singular trajectories in multi-input optimal problems. Application to controlled mechanical systems, J. Dynam. Control Systems, 9 (2003), pp. $73-88$.

[10] P. E. Crouch, Spacecraft attitude control and stabilization: Applications of geometric control theory to rigid body models, IEEE Trans. Automat. Control, 29 (1984), pp. 321-331.

[11] T. I. Fossen, Guidance and Control of Ocean Vehicles, Wiley, New York, 1994.

[12] I. I. Gihman, Concerning a theorem of N. N. Bogolyubov (Russian), Ukrain. Mat. Ž., 4 (1952), pp. 215-219.

[13] J. Kurzweil and Z. Vorel, Continuous dependence of solutions of differential equations on a parameter (Russian), Czechoslovak Math. J., 82 (1957), pp. 568-583.

[14] H. LAmb, Hydrodynamics, 6th ed., Cambridge University Press, Cambridge, 1932. 
[15] N. E. Leonard, Mechanics and Nonlinear Control: Making Underwater Vehicles Ride and Glide, Proceedings of the 4th IFAC Nonlinear Control Design Symposium (NOLCOS), Enschede, The Netherlands, pp. 1-6, 1998.

[16] W. Liu and H. J. Sussmann, Continuous dependence with respect to the input of trajectories of control-affine systems, SIAM J. Control Optim., 37 (1999), pp. 777-803.

[17] S. P. Novikov and I. Shmeltser, Periodic solutions of Kirchhoff's equations for the free motion of a rigid body in a fluid and the extended theory of Lyusternik-Shnirel'man- Morse (LSM), I. Funct. Anal. Appl., 15 (1982), pp. 197-207. 